\title{
Contribuição ao Processo de Afiação de Ferramentas Monocortantes de Aço Rápido
}

\section{Contribution to Sharpening Operation of HSS Lathe Tool Bit}

\author{
Raphael Lima de Paiva ${ }^{1}$, Rosemar Batista da Silva ${ }^{1}$, Rosenda Valdés Arencibia ${ }^{1}$, \\ Rodrigo de Souza Ruzzi ${ }^{2}$, Eduardo Carlos Bianchi ${ }^{2}$, Paulo Roberto de Aguiar $^{2}$
}

\author{
${ }^{1}$ Universidade Federal de Uberlândia, Faculdade de Engenharia Mecânica, Campus Santa Mônica, Uberlândia - MG, \\ Brasil \\ e-mail: raphael.paiva@ufu.br; rosemar.silva@ufu.br; rosenda.arencibia@ufu.br \\ ${ }^{2}$ Universidade Estadual Paulista - UNESP, Faculdade de Engenharia de Bauru Av. Luiz Edmundo Carrijo Coube, 14-01, \\ Bauru - SP, Brasil \\ e-mail: rodruzzi@gmail.com; bianchi@feb.unesp.br; aguiarpr@feb.unesp.br
}

\begin{abstract}
RESUMO
Apesar do grande desenvolvimento de ferramentas com insertos de metal duro, material cerâmico e outros nas últimas três décadas, algumas das ferramentas de corte ainda necessitam ser reafiadas após a perda do corte por meio do processo de afiação com rebolos abrasivos. A afiação tem como objetivo gerar as arestas de corte de ferramentas novas ou restabelecer as arestas de corte de ferramentas desgastadas e, para que este processo seja eficiente, ela dependerá da seleção correta dos parâmetros de corte, da máquina ferramenta e habilidade do operador. Se neste processo um parâmetro de corte não for corretamente selecionado a geometria poderá ser comprometida e, no pior dos casos, a peça poderá sofrer queima. A queima leva, na maioria dos casos, à perda da dureza da ferramenta, propriedade essencial para que a ferramenta consiga resistir aos esforços durante a usinagem como, por exemplo, quando uma ferramenta monocortante de aço rápido é empregada no processo de torneamento cilíndrico de aços ao carbono. A fim de estudar o processo de afiação deste tipo de ferramenta, esta pesquisa aborda o procedimento de afiação com quatro diferentes valores de profundidade de corte, considerado um dos principais parâmetros que afeta a qualidade da geometria gerada. Utilizou-se um rebolo do tipo copo reto de óxido de alumínio branco, típico para esta operação. O material da peça foi o aço rápido (HSS) com dureza de $62 \pm 2$ HRC. As variáveis de saída foram os ângulos de saída, de posição principal e de posição secundário, que foram medidos com o auxílio de um projetor de perfil e um transferidor universal (apenas para o ângulo de saída). Além disso, a incerteza de medição foi calculada para caracterizar a dispersão dos valores atribuídos a cada ângulo medido. Os resultados mostraram que houve variação nas medidas dos ângulos em função da penetração de trabalho, como esperado, e que a maior exatidão dos ângulos gerados foi obtida após a profundidade de corte igual a 0,06 mm. Não houve formação de rebarba e nem a presença de queima das superfícies usinadas.
\end{abstract}

Palavras-chave: usinagem, afiação de ferramentas, ângulos, profundidade de corte, incerteza de medição.

\section{ABSTRACT}

Despite of technological advances in developments of cutting tools such as carbide inserts, ceramic materials and others in the last three decades, some of cutting tools still needs to have their cutting geometry restored due to wear through the sharpening operation with abrasive wheels. Sharpening aims to provide shape either to new cutting tools and worn ones. The efficiency of this operation depends on the correct selection of cutting parameters, machine tool and operator's ability. In this process, if an improper cutting parameter is selected, the workpiece geometry can be compromised and, at the worst-case scenario, surface burning can occur, thereby leading to reduction of tool hardness. Tool hardness and strength are essential to withstand cutting forces during machining, for example, when a lathe tool bit is employed for cylindrical turning of a mild steel. In this context, this work presents an experimental study on the sharpening operation of HSS lathe tool bit using four different values of depth of cut. The grinding wheel employed was a straight cup white 
aluminium oxide, which is highly recommended for such operation. The workpiece material was the high speed steel (HSS) with hardness of $62 \pm 2$ HRC. The output parameters evaluated were the values of the rake angle, side cutting edge angle and end cutting edge angle, that were all measured with aid of a profile projector and a universal protractor (only for the rake angle). All the experimental trials were replicated once and the angles were measured five times. Statistical analyses of the results were performed to evaluate the statistical significance of comparisons and the measurement uncertainty was calculated. The results showed that all the angles were affected by the depth of cut, as expected, and the highest angles accuracy was obtained after machining with a depth of cut of $0.06 \mathrm{~mm}$. No burrs and burning of the machined surfaces were observed.

Keywords: machining, sharpening cutting tools, angles, depth of cut, measurement uncertainty.

\section{INTRODUÇÃO}

É natural que as ferramentas de corte se desgastem com o tempo de forma a terem alteradas a geometria da cunha de corte. Dependendo das condições de trabalho das ferramentas, junto com o desgaste podem também surgir trincas e mudanças nas propriedades mecânicas. Esse desgaste pode gerar problemas como aquecimento excessivo, aumento do esforço de corte, pobre acabamento superficial e perda de produtividade. YINGFEI et al. [1], por exemplo, ao realizarem o torneamento da liga Ti-6Al-4V com ferramenta de PCD, observaram um aumento de até $140 \%$ nos esforços de corte após um desgaste de flanco de 0,2 mm. Como soluções, podese efetuar a troca da ferramenta desgastada por uma nova ou restaurá-la através do processo de afiação. Definida como sendo o processo de conferir forma às arestas das ferramentas de corte novas ou de restaurar o corte ou o perfil de ferramentas desgastadas pelo uso, o processo de afiação com abrasivos destaca-se pela sua importância em torneadoras, laboratórios de usinagem, ferramentarias e outros locais fabris onde a utilização de bits e bedames é muito comum, visto que por meio desse processo é possível formar a geometria de corte dessas ferramentas (no caso de bits e bedames novos) ou restabelecer a geometria da aresta cortante das ferramentas desgastadas. Dessa forma, o processo de afiação contribui para evitar o descarte desnecessário de ferramentas desgastadas que, após serem afiadas, podem ser novamente utilizadas.

Embora a aquisição de uma nova ferramenta para substituir àquela já desgastada seja uma solução rápida e prática, a afiação (restauração dessa ferramenta desgastada) é uma grande oportunidade para reduzir custos, tendo em vista que uma ferramenta recondicionada pode-se alcançar o mesmo rendimento de uma ferramenta nova com custo menor, de até 10\% [2]. MOURA e MACHADO [3] avaliaram o desempenho de ferramentas de metal duro reafiadas no fresamento do aço VP20ISOF (bastante empregado em aplicações diversas em moldes para transformação de plástico) em relação às ferramentas novas e concluíram que, para uma confiabilidade de 95\%, não houve diferença estatisticamente significativa entre as ferramentas reafiadas e as novas nas condições investigadas.

Diferente de processos de usinagem como torneamento, fresamento e furação, a afiação é um processo de usinagem que emprega rebolo abrasivo e, por isso, é classificada como um processo de usinagem por abrasão. Na usinagem por abrasão a remoção de material se dá pela presença de grãos abrasivos não metálicos de elevada dureza que não possuem geometria definida, os quais podem ser unidos por um ligante ou soltos, que são colocados em contato com o material da peça [4]. Devido ao fato dos grãos que promovem o corte na usinagem por abrasão possuírem arestas pequenas que implicam em baixos valores de penetração de trabalho, este processo de usinagem é capaz de assegurar a produção de componentes com desvios dimensionais e geométricos inferiores (maior exatidão) àqueles obtidos em operações que utilizam ferramentas de corte com geometria definida [5].

A correta seleção dos parâmetros de corte na afiação irá determinar a eficiência do processo, principalmente na qualidade da geometria gerada. E o primeiro passo é a seleção do rebolo. Na afiação de ferramentas de aço rápido são recomendados rebolos abrasivos convencionais a base de óxido de alumínio $\left(\mathrm{Al}_{2} \mathrm{O}_{3}\right)$, enquanto que para as ferramentas de corte de metal duro empregam-se rebolos de carbeto de silício ( $\mathrm{SiC})$. O processo de afiação também pode ser realizado com ferramentas superabrasivas, como o CBN e diamante, mas devido ao elevado custo, o emprego deles só é justificado em máquinas ferramentas para produção em série e que operam em elevadas velocidades de corte, bem superiores àquelas empregadas com rebolos convencionais. A menor dureza apresentada pelo óxido de alumínio implica numa maior resistência à fratura ou fragmentação devido ao impacto ou quando do engajamento do rebolo com a peça, justificando, por estas características, o uso dele em operações de desbaste/semi-acabamento em materiais para ferramentas não tão duros como, por exemplo, aços rápidos [6].

Sabe-se que a geometria de uma ferramenta de corte exerce grande influência no desempenho da usinagem, e por melhor que seja o material da ferramenta, se a sua geometria não for preparada adequadamente, 
o desempenho do processo poderá ser comprometido [5]. O desempenho de uma ferramenta afiada é determinado pela sua capacidade de cortar adequadamente, sem ocorrência de perda de dureza ou trincas. E esta capacidade de corte está relacionada com a qualidade da geometria, ou seja, com a superfície usinada e valores dos ângulos gerados conforme projeto. No caso específico de ferramentas de aço rápido (HSS), de acordo com SARAVANAPRIYAN et al. [7], o desempenho da ferramenta depende principalmente das condições de corte e da geometria da ferramenta.

A geometria de uma ferramenta de corte é formada por diversos ângulos, que são resultado do encontro de três superfícies: principal de folga $(\mathrm{A} \alpha)$, secundária de folga $\left(\mathrm{A}^{\prime} \alpha\right)$ e de saída $(\mathrm{A} \gamma)$. Na Fig. 1 (a) são mostradas tais superfícies para o caso de uma ferramenta utilizada no processo de torneamento. Dessa forma, o processo de afiação, seja para gerar ou restaurar a aresta cortante, é feita somente nessas superfícies. Do encontro dessas três superfícies, seis ângulos merecem destaque, os medidos no plano de referência (ângulo de posição principal - $\chi_{r}$, ângulo de posição secundário - $\chi^{\prime}{ }_{r}$ e ângulo de ponta - $\xi_{\mathrm{r}}$ ) e os medidos no plano ortogonal (ângulo de folga - $\alpha_{0}$, ângulo de saída - $\gamma_{0}$ e ângulo de cunha - $\beta_{0}$ ). Nas Fig. 1 (b) e (c) são mostrados tais ângulos para o caso de uma ferramenta para torno.

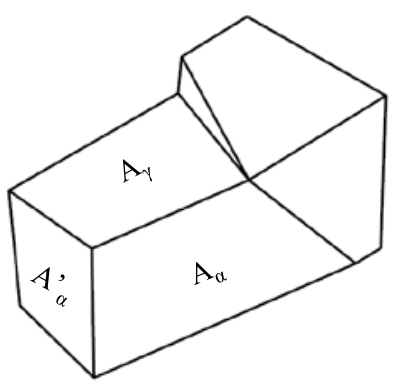

(a)

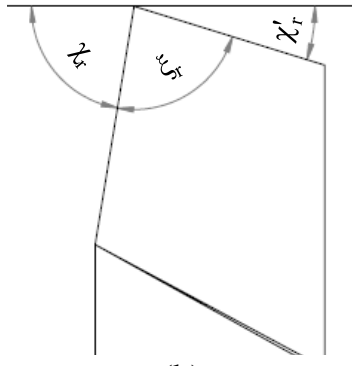

(b)

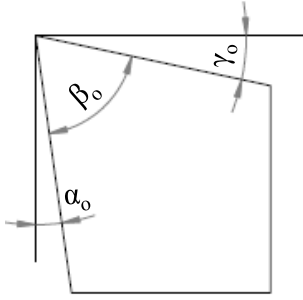

(c)

Figura 1: Ferramenta para torno com suas três superfícies principais (a) e vista dos planos de referência (b) e ortogonal (c) com seus principais ângulos.

A qualidade do processo de afiação pode ser quantificada, dentre outras formas, por meio da exatidão da geometria gerada, isto é, dado um valor de referência para cada um dos seis ângulos mostrados nas Fig. 1 (b) e (c), a afiação da ferramenta seria perfeita se os ângulos formados tivessem exatamente o mesmo valor dos de referência.

Neste sentido, este trabalho apresenta os resultados do processo de afiação de bits de aço rápido com quatro diferentes valores de profundidade de corte. Este parâmetro foi selecionado por ser relatado na literatura específica como sendo o mais influente na qualidade da geometria gerada. Como variáveis de saída, foram investigados os valores dos ângulos de saída, de posição principal e de posição secundário. Além disso, foi calculada a incerteza da medição para estes ângulos, afim de que verificar a dispersão dos valores atribuídos a cada ângulo.

\section{MATERIAIS E MÉTODOS}

Os ensaios de afiação foram realizados em uma máquina afiadora universal AtlasMaq, modelo PP-6025Q com $0,560 \mathrm{~kW}$. O rebolo utilizado foi o de óxido de alumínio branco $\left(\mathrm{Al}_{2} \mathrm{O}_{3}\right)$ do tipo copo reto, designação 6A AA46 K8V40W e dimensões de 101,60 mm de diâmetro externo, 50,80 mm de largura e 31,75 mm de diâmetro interno, do fabricante Carborundum. O sentido de rotação adotado foi o anti-horário.

Foram utilizados 4 bits de HSS (12\% Co) na forma de barra retangular com dimensões de 101,6 mm de comprimento e 9,525 mm de seção quadrada. Estas barras foram seccionadas ao meio, totalizando, portanto, oito amostras. Os ângulos de referência selecionados foram: ângulo de saída $\left(\gamma_{0}\right)$ : $12^{\circ}$; ângulo de posição principal $\left(\chi_{\mathrm{r}}\right): 75^{\circ}$ e ângulo de posição secundário $\left(\chi_{\mathrm{r}}^{\prime}\right): 15^{\circ}$.

Neste processo, em geral a velocidade de corte do rebolo é constante e o avanço da mesa é realizado manualmente. Os parâmetros de corte selecionados para os experimentos foram: velocidade de corte (Vs) igual $30 \mathrm{~m} / \mathrm{s}$ e avanço (f) manual (cerca de $1000 \mathrm{~mm} / \mathrm{min}$ ) que foram mantidos constantes para todos os 8 ensaios. Foram empregados quatro valores de profundidade de corte $\left(a_{p}\right): 0,04 \mathrm{~mm}, 0,06 \mathrm{~mm}, 0,08 \mathrm{~mm}$ e 0,10 mm. Para cada condição de corte utilizada foram afiadas duas ferramentas identificadas como "Ferramenta 1" e "Ferramenta 2”. Todos os ensaios foram realizados na presença de fluido de corte emulsionável, Satisol 1, 
do fabricante Ipiranga, a uma proporção de 1:19, com concentração $\cong 5 \%$ que foi verificada antes de cada ensaio com o auxílio de um refratômetro modelo N1, Atago, com Brix de 0-32\%. Na Tabela 1 são listadas as condições de corte utilizadas neste trabalho.

Para obter a geometria completa, foram formadas as três superfícies da ferramenta: superfície principal de folga, superfície secundária de folga e superfície de saída. Para cada uma dessas superfícies, foi necessário um ajuste diferente na máquina, conforme mostrado na Fig. 2. Este ajuste é realizado por uma combinação entre a morsa giratória (responsável pelo posicionamento angular do bit no plano de referência) e o eixo principal do rebolo (responsável pelo posicionamento angular do bit no plano ortogonal). O ângulo de folga foi selecionado e gerado com valor de $8^{\circ}$, embora não tenha sido o foco deste trabalho. É importante salientar que os valores de referência para todos os ângulos foram selecionados com base na literatura, considerando a usinagem de aço comum ao carbono com ferramentas de aço rápido [6].

Tabela 1: Condições de corte utilizadas neste trabalho.

\begin{tabular}{l|l}
\hline REBOLO & $\mathrm{Al}_{2} \mathrm{O}_{3}$ - Tipo copo reto \\
& Especificação 6A AA46 K8V40W \\
\hline PEÇA & Bits de HSS (12\% Co) - 62 HRC \\
\hline ÂNGULOS DE REFERÊNCIA & $\gamma_{\mathrm{o}}=12^{\circ}$ \\
& $\chi_{\mathrm{r}}=75^{\circ}$ \\
& $\chi_{\mathrm{r}}^{\prime}=15^{\circ}$ \\
\hline VELOCIDADE DE CORTE $(\mathbf{V s})[\mathrm{m} / \mathrm{s}]$ & 30 \\
\hline VELOCIDADE DE AVANÇO $[\mathrm{mm} / \mathrm{min}]$ & 1000 \\
\hline PROFUNDIDADE DE CORTE $\left(\mathbf{a}_{\mathbf{p}}\right)[\mathrm{mm}]$ & 0,$04 ; 0,06 ; 0,08$ e 0,10 \\
\hline FLUIDO DE CORTE & Emulsionável, Satisol 1, Ipiranga \\
& Concentração 5\%, vazão de $6 \mathrm{~L} / \mathrm{min}$ \\
\hline
\end{tabular}

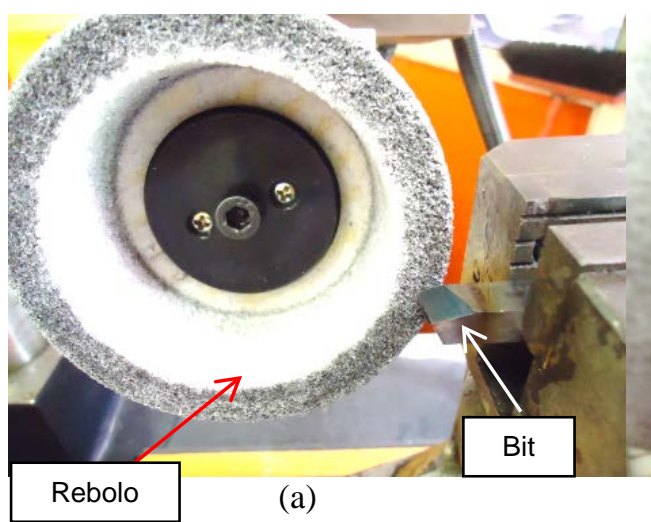

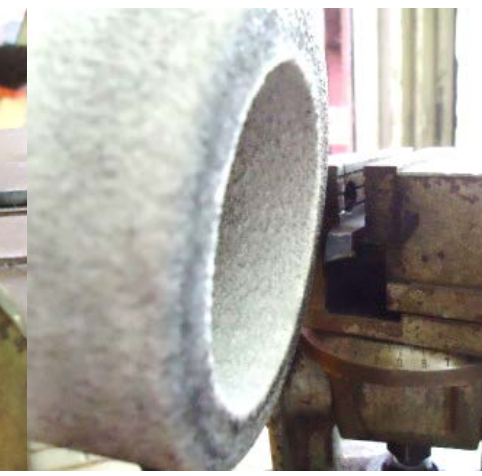

(b)

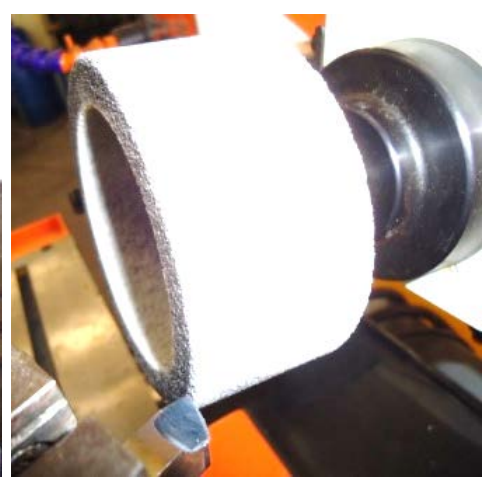

(c)

Figura 2: Disposição do rebolo-morsa-bit para a geração das superfícies: (a) de saída; (b) principal de folga; (c) secundária de folga.

O projetor de perfil MP320, Carl Zeiss, JENA, com resolução de 2', foi utilizado para a medição dos ângulos de posição principal e secundário. Durante a medição foi utilizada uma lente de 20x. Para a medição do ângulo de saída da ferramenta, foi utilizado um transferidor universal do fabricante Mitutoyo, devido à dificuldade de medição para tal ângulo no projetor de perfil. Este equipamento possui resolução de $5^{\prime}$.

As medições dos ângulos foram efetuadas a temperatura ambiente controlada de $(20 \pm 1){ }^{\circ} \mathrm{C}$, conforme recomendado pela norma ABNT NBR NM ISO 1:1997 [8]. Para monitoramento da mesma foi utilizado um termo higrômetro digital com resolução de $0,1^{\circ} \mathrm{C}$ e faixa nominal de $(-20$ a 60$){ }^{\circ} \mathrm{C}$. Este equipamento possui certificado de calibração N. R4996/13, emitido pelo Laboratório de Temperatura e Umidade da Elus Instrumentação. Para a temperatura a incerteza expandida é de $0,3{ }^{\circ} \mathrm{C}$ para k igual a 2,00 e infinitos graus de liberdade. Foi aguardado um período de oito horas até as peças e todos os equipamentos e dispositivos de medição entrassem em equilíbrio térmico com o ambiente. 


\section{RESULTADOS E DISCUSSÕES}

Os valores dos ângulos medidos são apresentados na Figura 3, em forma de gráficos, juntamente com seus respectivos valores de referência (VR). Para cada profundidade de corte $\left(a_{p}\right)$ foram geradas duas ferramentas diferentes (Ferramenta 1 e Ferramenta 2). Por sua vez, os valores médios dos ângulos gerados nas ferramentas 1 e 2, em função de cada valor de profundidade de corte, são apresentados na Tabela 2.

Nos gráficos da Figura 3 é possível observar que há diferenças entre os valores dos ângulos obtidos durante a afiação e os valores de referência adotados. Para o ângulo de posição principal observa-se que há uma tendência para a obtenção de ângulos menores que o valor de referência, enquanto que para os ângulos de posição secundários e de saída os valores obtidos são sempre maiores que a referência. Destaca-se que são observados erros sistemáticos da ordem de até $3^{\circ}$, indicativo de pouca exatidão. A justificativa para este fato pode estar em que a afiação é um o processo muito sensível a vários parâmetros de corte, como também depende da habilidade e capacitação do operador.

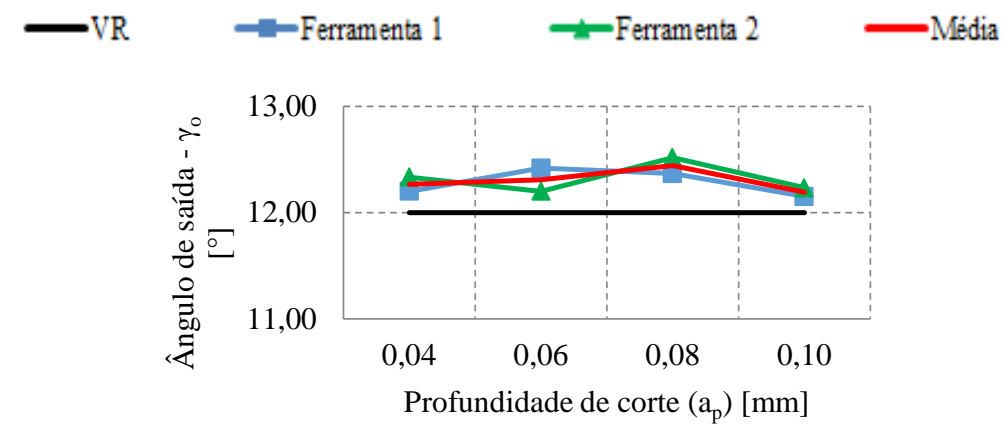

(a)

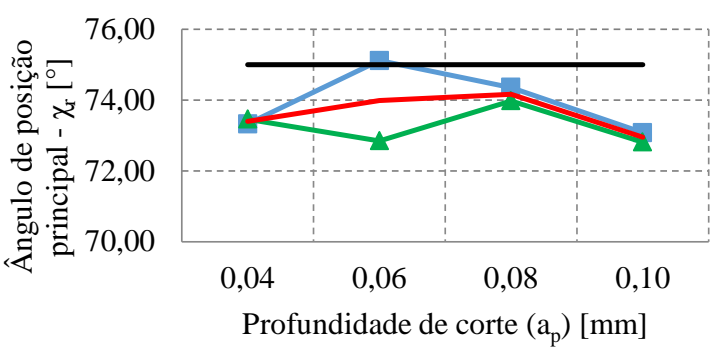

(b)

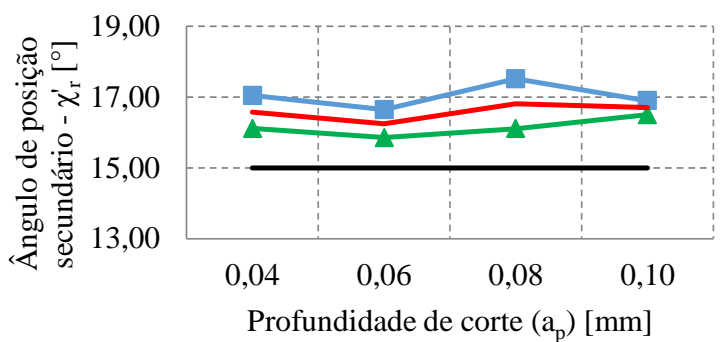

(c)

Figura 3: Valores dos ângulos medidos após processo de afiação.

Na Fig. 3 (a) ainda pode-se observar para o ângulo de saída $\left(\gamma_{0}\right)$ que os valores dos ângulos são maiores que o valor de referência para todas as condições, sendo que a maior diferença é, em média, de 25 ' para a profundidade de corte de $0,08 \mathrm{~mm}$. Neste caso, a melhor exatidão foi observada para a profundidade de 0,10 mm (erro sistemático de 10').

Tabela 2: Média dos ângulos medidos.

\begin{tabular}{|c|c|c|c|}
\hline & \multicolumn{3}{|c|}{ ÂNGULOS } \\
\hline & SAÍDA $\left(\gamma_{0}\right)$ & POSIÇÃO PRINCIPAL $\left(\gamma_{r}\right)$ & POSIÇÃO SECUNDÁRIO $\left(\chi_{\mathrm{r}}^{\prime}\right)$ \\
\hline$a_{a_{p}(\mathrm{~mm})} V R$ & $12^{\circ}$ & $75^{\circ}$ & $15^{\circ}$ \\
\hline 0,04 & $12^{\circ} 15^{\prime}$ & $73^{\circ} 24^{\prime}$ & $16^{\circ} 34^{\prime}$ \\
\hline 0,06 & $12^{\circ} 20^{\prime}$ & $73^{\circ} 58^{\prime}$ & $16^{\circ} 14^{\prime}$ \\
\hline 0,08 & $12^{\circ} 25^{\prime}$ & $74^{\circ} 10^{\prime}$ & $16^{\circ} 48^{\prime}$ \\
\hline 0,10 & $12^{\circ} 10^{\prime}$ & $72^{\circ} 56^{\prime}$ & $16^{\circ} 42^{\prime}$ \\
\hline
\end{tabular}


Ao analisar a Tabela 2 em relação aos ângulos de posição principal $\left(\chi_{\mathrm{r}}\right)$ e secundário $\left(\chi_{\mathrm{r}}^{\prime}\right)$ [Fig. 3 (b) e (c)], observa-se que o valor mais discrepante em relação ao de referência foi, em média, de $1^{\circ} 29^{\prime}\left(\chi_{r}^{\prime}, a_{p}=\right.$ $0,08 \mathrm{~mm}$ ). $\mathrm{O}$ ajuste da máquina afiadora para propiciar a usinagem das duas superfícies e, consequentemente gerar estes dois ângulos (de posição principal e secundário), é bem semelhante e depende apenas da graduação da morsa que está sobre a mesa da máquina.

Em relação ao ângulo de posição principal $\left(\chi_{\mathrm{r}}\right)$ [Fig. 3 (b)], a pior exatidão foi encontrada para a profundidade de corte de $0,10 \mathrm{~mm}$. Neste caso, o erro sistemático assume $3^{\circ} 4^{\prime}$. Por sua vez, para a profundidade de corte de $0,08 \mathrm{~mm}$ foi obtido o menor erro sistemático (50’) e a maior exatidão. Para o ângulo de posição secundário $\left(\chi_{\mathrm{r}}^{\prime}\right)$ [Fig. $3(\mathrm{c})$ ] a profundidade de corte de $0,06 \mathrm{~mm}$ forneceu a maior exatidão (erro sistemático de $46^{\prime}$ ) enquanto que a profundidade de corte de $0,08 \mathrm{~mm}$ gerou um erro sistemático de 12 ' e a pior exatidão.

É sabido da literatura específica que a profundidade de corte exerce influência direta na qualidade superficial das superfícies usinadas. Quanto menor for o valor da profundidade de corte, mantendo-se constantes o avanço e a velocidade do rebolo, menor é o esforço da máquina e menor é a penetração de cada grão abrasivo na superfície da peça. MARINESCU et al. [9] afirmaram que a altura total do perfil de rugosidade (parâmetro de rugosidade Rt) é dependente primeiramente da velocidade do rebolo e em seguida da penetração de trabalho, de forma que pode ser estabelecida a relação Rt $\sim \sqrt{\mathrm{a}_{\mathrm{e}}}$. Seria de se esperar a correlação direta entre qualidade da superfície (rugosidade) e a qualidade da superfície e aresta geradas por afiação para cada combinação de superfícies da ferramenta, mas neste trabalho e nas condições investigadas esta correlação não foi observada.

Ao analisar a influência da profundidade de corte na exatidão dos ângulos gerados no processo, percebe-se que, de forma geral, os bits afiados com $\mathrm{a}_{\mathrm{p}}=0,06 \mathrm{~mm}$ são aqueles que apresentaram melhor exatidão, exceto para o $\chi_{\mathrm{r}}$ da Ferramenta 2, cujo valor medido apresentou um desvio acima de $2^{\circ}$ em relação ao valor de referência. Entretanto, ao analisar o valor médio para $\chi_{\mathrm{r}}$ na Tab. 2, observa-se que esse desvio diminui, e que a diferença entre o valor médio mais próximo do de referência $\left(a_{p}=0,08 \mathrm{~mm}\right)$ e o valor médio para $a_{p}=$ $0,06 \mathrm{~mm}$ é de apenas 12 . Ressalta-se que à medida que se aumenta o valor de $\mathrm{a}_{\mathrm{p}}$, seja para $0,08 \mathrm{~mm}$ ou 0,10 $\mathrm{mm}$, os ângulos gerados se afastam do valor de referência. Uma explicação para os resultados não satisfatórios para $\mathrm{a}_{\mathrm{p}}=0,04 \mathrm{~mm}$ pode ser o fato de que para pequenos valores de $\mathrm{a}_{\mathrm{p}}$ as arestas dos grãos na superfície do rebolo não tenham penetrado eficientemente a fim de garantir a espessura de corte mínima necessária para a formação de cavaco, mas prevalecendo as etapas de deformação plástica e riscamento, ou seja, não foram agressivos suficientemente para remoção de cavaco. Vale ressaltar que a etapa de riscamento pode também ser responsável pela arrancada de material, que muitas vezes pode implicar em acúmulo de material nas bordas da peça deixadas pelos grãos. Para os elevados valores de profundidade de corte, já eram esperados tais resultados uma vez que resultam em acréscimo das forças de corte que, por sua vez, causam deformações entre peça e ferramenta.

Na Figura 4 são apresentadas as imagens das ferramentas com suas arestas após afiação com diferentes profundidades de corte (vista do plano de referência). Observa-se que não houve formação de rebarba e nem a presença de queima da superfície e consequentemente da aresta de corte. As manchas de coloração escura presentes nas ferramentas são marcas provenientes da formação de óxido da combinação da temperatura de usinagem e fluido de corte empregado.

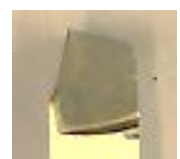

(a)

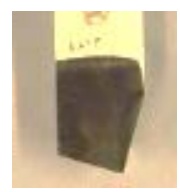

(e)

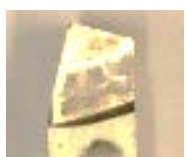

(b)

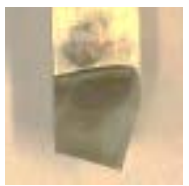

(f)

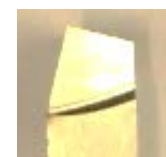

(c)

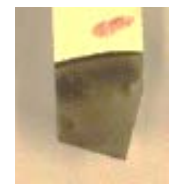

(g)

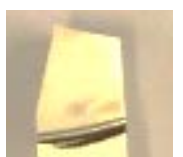

(d)

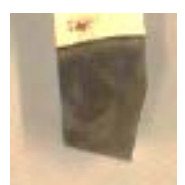

(h)

Figura 4: Imagens das ferramentas (plano de referência) após processo de afiação. (a) Ferramenta 1, $\mathrm{a}_{\mathrm{p}}=0,04 \mathrm{~mm}$; (b) Ferramenta 1, $a_{p}=0,06 \mathrm{~mm}$; (c) Ferramenta 1, $a_{p}=0,08 \mathrm{~mm}$; (d) Ferramenta 1, $\mathrm{a}_{\mathrm{p}}=0,10 \mathrm{~mm}$; (e) Ferramenta 2, $\mathrm{a}_{\mathrm{p}}=0,04$ mm; (f) Ferramenta 2, $a_{p}=0,06$ mm; (g) Ferramenta 2, $a_{p}=0,08$ mm; (h) Ferramenta 2, $a_{p}=0,10 \mathrm{~mm}$. 
Na Figura 5 são mostrados os valores da incerteza expandida associados à medição dos diferentes ângulos para todas as condições investigadas, onde:

- $\quad \gamma_{0}$ F1 - média para o ângulo de saída da Ferramenta 1;

- $\quad$ o F2 - média para o ângulo de saída da Ferramenta 2;

- $\quad$ r F1 - média para ângulo de posição principal da Ferramenta 1;

- $\quad$ xr F2 - média para o ângulo de posição principal da Ferramenta 2;

- $\quad$ ’r F1 - média para o ângulo de posição secundário da Ferramenta 1;

- $\quad \chi ’$ 'r F2 - média para o ângulo de posição secundário da Ferramenta 2.

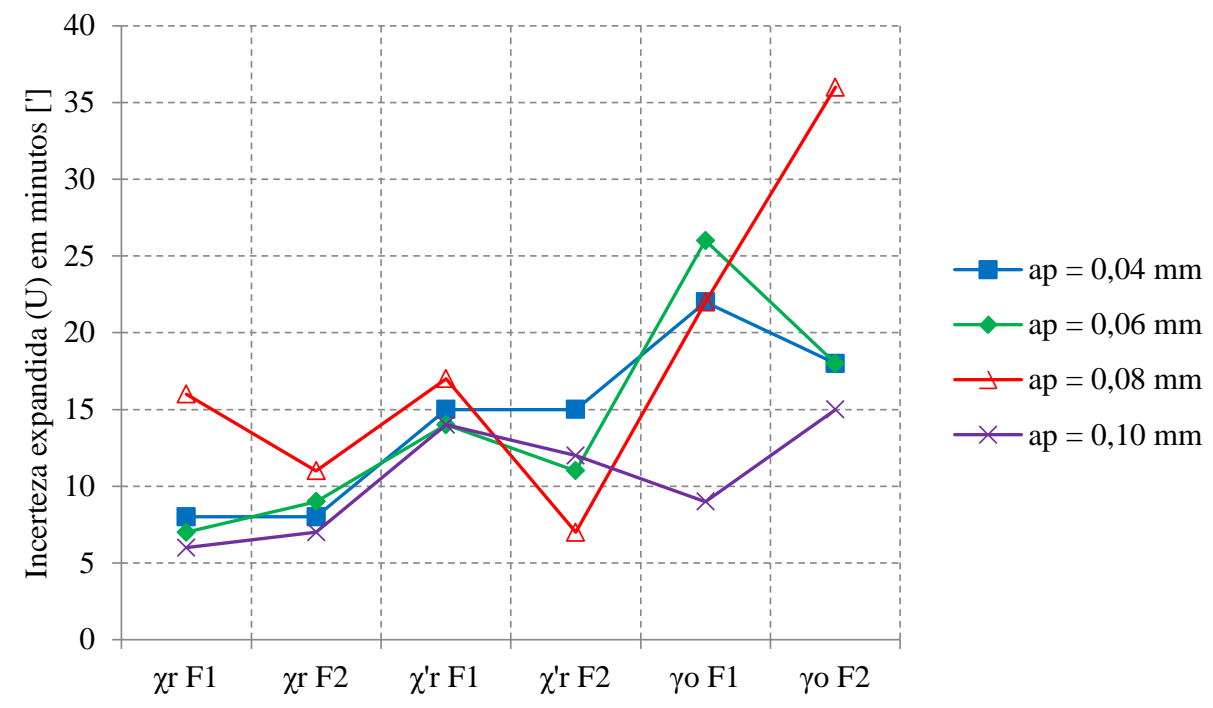

Figura 5: Incerteza expandida associada à medição do ângulo da geometria do bit.

Observa-se da Figura 5 que os valores de incerteza expandida associados à medição dos diferentes ângulos avaliados são pequenos, variando entre $7^{\prime}$ e $37^{\prime}$. Estes valores podem ser considerados adequados para a exatidão requerida na medição. A variável que mais contribuiu para a incerteza final, em todos os casos, foi a variabilidade dos valores indicados pelo projetor de perfil.

\section{CONCLUSÕES}

As seguintes conclusões foram retiradas deste trabalho:

- Os ângulos de saída, de posição principal e de posição secundário sofreram influência da profundidade de corte. A eficiência na geração de cada ângulo testado implica na seleção de um valor específico de profundidade de corte.

- Os bits de HSS afiados com $\mathrm{a}_{\mathrm{p}}=0,06 \mathrm{~mm}$ apresentaram, para a metodologia aplicada a este trabalho, uma maior exatidão em relação aos outros valores de profundidade de corte. À medida que se aumentou o valor de $a_{p}$ para valores superiores a $0,06 \mathrm{~mm}$, os ângulos gerados se afastaram do valor de referência.

- A maior exatidão de resultados foi observada para o ângulo de saída;

- O cálculo da incerteza de medição expandida associada à medição dos ângulos indicou que os valores são considerados adequados para a exatidão requerida na medição;

- Não houve formação de rebarba e nem a presença de queima da superfície e consequentemente da aresta de corte após a operação de afiação nas condições de corte empregadas.

\section{AGRADECIMENTOS}

Os autores agradecem ao apoio do CNPq e da CAPES. Um dos autores agradece em especial à FAPEMIG pelo apoio financeiro via projeto PPM-VII, processo $\mathrm{n}^{\circ}$. 00265-13 como também à CAPES pelo apoio financeiro via bolsa de pós-doutorado, PNDP, cujas atividades foram desenvolvidas na FEB-UNESP-BAURU. 


\section{BIBLIOGRAFIA}

[1] YINGFEI, G., JIUHUA, X., HAIXIANG, H., “Tool wear during high speed turning in situ TiCp/TiBw hybrid reinforced Ti-6Al-4V matrix composite”, Chinese Journal of Aeronautics, v.29, n.5, Jan. 2016.

[2] WAGNER, C., Redução de custos através da afiação de ferramentas de corte: o caso de uma empresa do setor metal - mecânico, Trabalho de Conclusão de Curso, UDESC, Santa Catarina, PR, Brasil, 2008.

[3] MOURA, R.R., MACHADO, A.R., "Machining of VP20ISOF steel with resharpened carbide tools in end milling”, In: Proceeding of the ASME 2013 International Manufacturing Science and Engineering Conference, Madison, Wisconsin, USA, June 2013.

[4] MALKIN, S., Grinding technology: theory and applications of machining with abrasives. England, Ellis Horwood Limited, 1989.

[5] MACHADO, A.R., COELHO, R.R., ABRÃO, A.M., et al., Teoria da Usinagem dos Materiais, 2 ed., São Paulo, Edgard Blücher, 2011.

[6] NUSSBAUM, G. C., Rebolos e abrasivos: Tecnologia básica, v. 1, São Paulo, Ícone, 1988.

[7] SARAVANAPRIYAN, S.N., VIJAYARAGHAVAN, L., KRISHNAMURTHY, R., "Significance of grinding burn on high speed steel tool performance”, Journal of Materials Processing Technology, v. 134, n. 2, pp. 166-173, Mar. 2003.

[8] ABNT NBR NM ISO 1:1997, Temperatura padrão para medições industriais de comprimento, Associação Brasileira de Normas Técnicas, 1997.

[9] MARINESCU, I.D., ROWE, W.B., DIMITROV, B., INASAKI, I., Tribology of abrasive machining processes, 1 ed., New York, William Andrew Inc., 2004. 